\title{
AN EXAMINATION OF FORWARD VOLATILITY
}

\author{
Ray Popovic \\ David Goldsman \\ School of Industrial and Systems Engineering \\ Georgia Institute of Technology \\ Atlanta, GA 30332, U.S.A.
}

\begin{abstract}
This paper investigates the adequacy of various principal components (p.c.) approaches as data reduction schemes for processing contingent claim valuations on baskets of equities. As a general proposition we are interested in discovering possible features and rules-of-thumb for the applicability of p.c. techniques. In particular, what accuracy does one lose in valuation-hedging schemes as the dimensionality of the p.c. space is reduced? We also have an interest in validating the posted "stylized" facts of implied volatility as they apply to our data sets.
\end{abstract}

\section{INTRODUCTION}

Early observation in organized options markets of the Black and Scholes (1973) and Merton (1971) valuation formula dedicated to claims with the same underlying, but with differing strike and expiry dates, indicated that the key constant volatility assumption of the standard valuation formula is incorrect - volatility is itself varying! A practical fix to this dilemma is to ignore it and quote an option's value in terms of implied sigma. In fact, once one gets used to it, quoting value in "sigma" units is more intuitive. Quoting relative value in terms of implied sigma works reasonably well when there is a single underlying factor driving the price of the contingent claim, or if there exists a claimdependent reduction converting several underlying driving forces to one, e.g., a Margrabe (1978) exchange-type option. The reason is that time until expiry and strike price are related to option premium nonlinearly. Consequently, it is difficult to discern if a particular premium, associated with some expiry date and strike price, is out of sync with the rest of the premia in the option chain. On the other hand, if a particular implied volatility is an outlier, it is more likely to be evident vis-a-vis the volatility values in the remaining links of the chain. In turn, this may indicate an exploitable profit opportunity.
We study the implications of varying volatility on vanilla and exotic option valuations. Since closed-form valuation formulas are scarce for anything but standard European option types, simulation will be the method of choice for implementing pricing. In order to calibrate our simulations, we use daily option chain data, e.g., entries for best bid and best ask quotes, the spectrum of "Greeks", volume, open interest, etc., for the time period 07/01/02 - 08/29/03, collected by Ivy DB OptionMetrics (Wharton Research Data Services). Since the use of all average and forward volatility data series for each of the underlying equity option chains can be onerous, we utilize the so-called common p.c. approach as a data reduction method. The hypothesis is that there exist common features of volatility among, at least, some of the considered equity groups. In a recent paper of Cont, Da Fonesca, and Durrleman (2002), some representative features as applied to individual - not joint - equity indices are catalogued. Also, Fengler, Härdle, and Villa (2001) apply a common p.c. approach to DAX index options. Related study of stochastic volatility is pursued by Fouque, Papanicolaou, and Sircar (2000), but with regard to high frequency data.

In the following section dealing with background we introduce in a general way the data-driven problem. Section 3 motivates an example where the variability of volatility can matter. The last section provides some useful places where the type of analysis we pursue could be implemented.

\section{BACKGROUND}

Example data on a major index and five component equities are presented in Tables 1 and 2. In the language of the statistical packages R/S/S+ (R Development Core Team 2004, Ihaka and Gentleman 1996), the tables represent one frame (trading day) of a multiple frame data set. Table 1 documents the closing price on the date in question along with the immediate bounding strike values. In the table, $S$ denotes unit equity value, $X_{L}=$ the nearest quoted strike 
Table 1: Price, Lower and Upper Strike Value Data: August 26, 2003

\begin{tabular}{|c||c||c||c||c||c||c|}
\hline Company & NASDAQ $100 \equiv Q$ & Intel $\equiv I$ & Dell $\equiv D$ & Microsoft $\equiv M$ & Amgen $\equiv A$ & Home Depot $\equiv H$ \\
\hline$X_{L}$ & 32.00 & 27.50 & 30.00 & 25.00 & 65.00 & 30.00 \\
$S$ & 32.54 & 27.71 & 31.93 & 26.57 & 65.53 & 32.24 \\
$X_{U}$ & 33.00 & 30.00 & 35.00 & 27.50 & 70.00 & 35.00 \\
\hline
\end{tabular}

Table 2: Implied Volatility Data: August 26, 2003 (See Text for Key)

\begin{tabular}{|c|c|c|c|c|c|c|c|c|c|c|c|c|}
\hline & & & & & & & & & & & & \\
\hline Expiry & $\sigma_{Q}$ & $\sigma_{Q_{[\cdots]}}$ & $\sigma_{I}$ & $\sigma_{I_{[\cdot,]}}$ & $\sigma_{D}$ & $\sigma_{D_{[\cdots,]}}$ & $\sigma_{M}$ & $\sigma_{M_{[,, \cdot]}}$ & $\sigma_{A}$ & $\sigma_{A_{[, \cdot]}}$ & $\sigma_{H}$ & $\sigma_{H_{[\cdot, \cdot]}}$ \\
\hline 20-Sep-03 & 25.48 & 25.48 & 30.25 & 30.25 & 30.40 & 30.40 & 26.93 & 26.93 & 24.66 & 24.66 & 25.04 & 25.04 \\
\hline 18 -Oct-03 & 28.94 & 31.28 & 33.98 & 37.07 & 28.53 & 27.75 & 26.79 & 26.93 & 28.07 & 30.84 & 26.40 & 28.58 \\
\hline 22-Nov-03 & 27.76 & 27.03 & & & 30.09 & 32.07 & & & & & 26.69 & 27.24 \\
\hline 20-Dec-03 & 27.96 & 27.88 & & & & & & & & & & \\
\hline 17-Jan-04 & 27.56 & 26.89 & 33.18 & 33.12 & 30.06 & 30.45 & 27.17 & 27.41 & 27.96 & 27.94 & 27.85 & 28.26 \\
\hline 21-Feb-04 & & & & & 30.47 & 30.73 & & & & & 27.08 & 27.26 \\
\hline 20-Mar-04 & 27.50 & 26.08 & & & & & & & & & & \\
\hline 17-Apr-04 & & & 33.84 & 35.22 & & & 28.06 & 29.00 & 28.89 & 28.33 & & \\
\hline 22-Jan-05 & 26.70 & 26.33 & 33.97 & 34.09 & 31.18 & 31.28 & 29.51 & 30.35 & 28.66 & 29.00 & 28.94 & 28.96 \\
\hline 21-Jan-06 & 26.41 & 25.59 & 34.22 & 34.70 & 31.23 & 30.22 & 27.85 & 25.81 & 27.63 & 26.46 & 29.46 & 29.34 \\
\hline
\end{tabular}

value bounding $S$ from below, and $X_{U}=$ the nearest quoted strike value bounding $S$ from above.

Next, Table 2 exhibits succinctly the challenge of calibrating a valuation engine. In this table, $\sigma_{x}$ denotes the ( $a v$ erage) implied volatility of equity $x$ and $\sigma_{x_{[\cdot, \cdot]}}=($ marginal $)$ forward implied volatility of equity $x$ on time interval $[\cdot, \cdot]$, $x=Q, I, D, M, A, H$, where the equities are given in Table 1. The volatility entries are volume weighted - upper and lower strike bracket - of at-the-money implied postings. (Due to this averaging process, the two volatility series for each equity may violate slightly the relationship between the average and marginal functions, i.e., the when average is increasing [decreasing], the marginal value is above [below] the average value.)

In Table 2, there are ten expiry dates and six sets of implied volatility series. One may place this frame in the context of a ten by six factor model - a driving factor for each equity option and each future expiry date. Looking at the table one sees gaps in the data. This is a consequence of options existing on different expiry cycles. Also, for some thinly traded positions in an option chain, postings may not be available, or what is even worse, posted values may be inaccurate.

A typical method of calibration "to the market" is to minimize the sum of squares between observed values and theoretical valuations. Such methods prove to be intertemporally unstable. They fit the model parameters to the data of the day only to see parameter estimates grossly change when re-estimation takes place tomorrow (cf. Pelsser 2000 on interest rate derivatives). Furthermore, they fail to provide an intermediate to long term view on what a portfolio's value may experience if it is hedged daily transaction costs do impact portfolio wealth.

Principal components analysis is a method suited for dimensionality reduction (see the texts of Flury 1988 or Härdle and Simar 2003; also, the paper of Hsieh 2004). By appropriate rotation, i.e., by choosing advantageous linear combinations of expiry indexed implied volatility time series, it is possible to reduce the influence of the totality of the number of underlying driving factors to that of a few important ones. It is often the case that one to three principal components will explain $95 \%-99 \%$ of the variability in the joint time series of volatility.

Flury and Gautschi (1986) provide an algorithm for the construction of an orthogonal matrix $\Gamma$ such that for positive definite symmetric (p.d.s.) covariance matrices $\left\{\Sigma_{k}\right\}_{k=1}^{K}$, we have that $\Gamma^{T} \Sigma_{k} \Gamma=\Lambda_{k}$ is diagonal or nearly so for each $k$. For any p.d.s. matrix $\Sigma$, the construction of $\Gamma$ uses as a measure of deviation from diagonal the value of the function $\varphi(\Sigma)=|\operatorname{diag}(\Sigma)| /|\Sigma|$, where $|\Sigma|$ is the determinant of the p.d.s. matrix $\Sigma$. Clearly, the lower bound of 1 is achieved if, to start with, $\Sigma$ is diagonal. As they point out, this measure is suited for the analysis of the maximum likelihood problem associated with common p.c.

\section{SIMPLE EXOTICS}

Shortly we will provide an example valuation formula, in closed form, that illustrates the usefulness of pursuing data reduction techniques such as p.c. The following decomposition of an equity price modeled as geometric Brownian motion proves to be useful as motivation for the study 
of models where volatility varies. For any $\omega \in \Omega$, let $\left(W_{\omega}(t), t \in[0, T]\right)$ be a standard Brownian motion path, let $\left\{Z_{i}^{\omega}\right\}_{i=1}^{n}$, for some finite $n=1,2, \ldots$, be a sequence of i.i.d. standard Gaussian random variables each identified by the c.d.f. $\Phi(\cdot)$, and consider points in time such that $0 \equiv T_{0}<T_{1}<\cdots<T_{N-1}<T_{N} \equiv T$. Setting $\Delta T_{[i-1, i]}=T_{i}-T_{i-1}$, we know from elementary properties of Brownian integrals that for time-dependent deterministic functions $t \rightarrow \sigma(t)$,

$$
\begin{aligned}
\int_{T_{i-1}}^{T_{i}} \sigma(s) d W(s) & \sim \mathbf{N}\left(0, \int_{T_{i-1}}^{T_{i}} \sigma^{2}(s) d s\right) \\
& \equiv \mathbf{N}\left(0, \sigma_{[i-1, i]}^{2} \Delta T_{[i-1, i]}\right)
\end{aligned}
$$

where $\sigma_{[i-1, i]}$ is interpreted as average volatility over the time interval $\left[T_{i-1}, T_{i}\right]$ and is usually quoted on an annualized basis. It naturally follows that for any $n^{\star}>n$,

$$
\begin{aligned}
\mathbf{N}\left(0, \int_{T_{n}}^{T_{n^{\star}}} \sigma^{2}(s) d s\right) \sim \int_{T_{n}}^{T_{n^{\star}}} \sigma(s) d W(s) \\
=\sum_{i=n+1}^{n^{\star}} \int_{T_{i-1}}^{T_{i}} \sigma(s) d W(s) \\
\sim \mathbf{N}\left(0, \sum_{i=n+1}^{n^{\star}} \sigma_{[i-1, i]}^{2} \Delta T_{[i-1, i]}\right) .
\end{aligned}
$$

Equation (1) shows that for purposes of simulation at the time points $T_{0}, T_{1}, \ldots, T_{N}$, we have equality in distribution between the idealized Brownian process and the process constructed from the i.i.d. sequence of random variables $\left\{Z_{i}^{\omega}, i=1,2, \ldots\right\}$ :

$$
\int_{T_{n}}^{T_{n^{\star}}} \sigma(s) d W(s) \stackrel{d}{=} \sum_{i=n+1}^{n^{\star}} \sigma_{[i-1, i]} \sqrt{\Delta T_{[i-1, i]}} Z_{i-1}^{\omega} .
$$

Whence, if $r$ is the annualized constant interest rate, and $n=1,2, \ldots, N$, the money market standardized stock price process $\left(S^{*}(t) \equiv S(t) \exp (-r t), t \in[0, T]\right)$, under the risk neutral measure is modeled as geometric Brownian motion:

$$
\begin{aligned}
S_{\omega}^{*}\left(T_{n}\right)= & S_{0} \mathrm{e}^{-\frac{1}{2} \int_{0}^{T_{n}} \sigma^{2}(s) d s+\int_{0}^{T_{n}} \sigma(s) d W(s)} \\
\stackrel{d}{=} & S_{0} \exp \left\{-\frac{1}{2} \sum_{i=1}^{n} \sigma_{[i-1, i]}^{2} \Delta T_{[i-1, i]}\right\} \\
& \times \exp \left\{\sum_{i=1}^{n} \sigma_{[i-1, i]} \sqrt{\Delta T_{[i-1, i]}} Z_{i}^{\omega}\right\} \\
\stackrel{d}{=} & S_{\omega}^{*}\left(T_{n-1}\right) \exp \left\{-\frac{1}{2} \sigma_{[n-1, n]}^{2} \Delta T_{[n-1, n]}\right\} \\
& \times \exp \left\{\sigma_{[n-1, n]} \sqrt{\Delta T_{[n-1, n]}} Z_{n}^{\omega}\right\},
\end{aligned}
$$

where $\omega \in \Omega$ and $S_{0} \equiv S(0)$ is the known initial unit stock price. As required by arbitrage-free arguments (Harrison and Kreps 1979, Harrison and Pliska 1981), the above formula is of the form of an exponential martingale (e.g., Øksendal 2003).

With (3) in hand, we introduce a simple yet compelling illustration of a closed-form contingent claim pricing formula where forward volatility structure matters. We value a component of the ratchet call. This instrument, though simple in structure, indicates the importance of estimating volatility as precisely as possible. The forward start call option (e.g., Cvitanić and Zapatero 2004) is, for each $n=$ $1,2, \ldots, N$, and for some $n^{\star}$ such that $n^{\star}>n$, defined by its value at time instant $t=T_{n^{\star}}$ to be $V\left(T_{n^{\star}}\right) \equiv \max \left\{S\left(T_{n^{\star}}\right)-\right.$ $\left.X\left(S\left(T_{n}\right)\right), 0\right\}$, where $X(\cdot)$ is the strike value, a known function at time $t=0$ of the unknown equity value at time $t=T_{n}$. This particular contingent claim depends on a subset of the joint distribution of stock path price outcomes. Namely, it depends on both the random strike price $X\left(S\left(T_{n}\right)\right)$ eventually observed at $t=T_{n}>T_{0}$ and the value the equity price achieves at option expiry time $t=T_{n^{\star}}>T_{n}$. The forward start option is a variant of the ratchet contingent claim often used in fixed income valuation. A ratchet call can be thought of as sequence of forward start options. Each successive forward start in the sequence commences on a date in the future when the preceding adjacent forward start expires. In the most basic setup, the strike price satisfies $X\left(S\left(T_{n}\right)\right) \equiv x S\left(T_{n}\right), x \in \mathbb{R}^{+}$. The closed-form solution is a variation on the Black-Scholes pricing formula:

$$
V(0)=S_{0} \Phi\left(z_{+}\right)-x S_{0} \mathrm{e}^{-r \Delta T_{\left[n, n^{\star}\right]}} \Phi\left(z_{-}\right),
$$

where

$$
z_{+} \equiv \frac{\ln \left(\frac{1}{x}\right)+\left(r+\frac{1}{2} \sigma_{\left[n, n^{\star}\right]}^{2}\right) \Delta T_{\left[n, n^{\star}\right]}}{\sigma_{\left[n, n^{\star}\right]} \sqrt{\Delta T_{\left[n, n^{\star}\right]}}}
$$

and

$$
z_{-} \equiv \frac{\ln \left(\frac{1}{x}\right)+\left(r-\frac{1}{2} \sigma_{\left[n, n^{\star}\right]}^{2}\right) \Delta T_{\left[n, n^{\star}\right]}}{\sigma_{\left[n, n^{\star}\right]} \sqrt{\Delta T_{\left[n, n^{\star}\right]}}} .
$$

Evidently, volatility enters in a forward way through the term $\sigma_{\left[n, n^{\star}\right]} \sqrt{\Delta T_{\left[n, n^{\star}\right]}}$. The more "missing" data that we can fill in with the aid of p.c. and common p.c. the better will be our approximation to the true volatility process, i.e., as $n_{\star} \rightarrow n$ we better approach instantaneous volatility.

We briefly sketch the derivation of (4) now. Given the time $t=0$ information set $\Im_{0}$ and applying the martingale property under the so-called usual risk-neutral measure to 
the process $\left(V(t) \mathrm{e}^{-r t}, t \in[0, T]\right)$, we obtain

$$
\begin{aligned}
& V(0)=E\left[\frac{V\left(T_{n^{\star}}\right)}{\mathrm{e}^{r T_{n^{\star}}}} \mid \Im_{0}\right] \\
& \quad=E\left[\max \left\{S^{*}\left(T_{n^{\star}}\right)-x S^{*}\left(T_{n}\right) \mathrm{e}^{-r \Delta T_{\left[n, n^{\star}\right]}}, 0\right\} \mid \Im_{0}\right] .
\end{aligned}
$$

From the definition of a ratchet call, and using the equity dynamics (3), we see that the constraint set over which averaging takes place - aside from sets of zero measure - satisfies:

$$
\begin{aligned}
\theta & =\left\{\omega \in \Omega: S_{\omega}\left(T_{n^{\star}}\right)>x S_{\omega}\left(T_{n}\right)\right\} \\
& =\left\{S_{\omega}^{*}\left(T_{n^{\star}}\right)>x S_{\omega}^{*}\left(T_{n}\right) \mathrm{e}^{\left.-r \Delta T_{\left[n, n^{\star}\right]}\right\}}\right. \\
& =\left\{Z_{\omega}^{\star}>\frac{\ln (x)-\left(r-\frac{1}{2} \sigma_{\left[n, n^{\star}\right]}^{2}\right) \Delta T_{\left[n, n^{\star}\right]}}{\sigma_{\left[n, n^{\star}\right]} \sqrt{\Delta T_{\left[n, n^{\star}\right]}}}\right\} .
\end{aligned}
$$

Substituting (3) into the second line of (5), letting $\frac{d \Phi(t)}{d t} \equiv$ $\phi(t)$, setting $\mathbf{1}_{\theta}$ as the indicator function of event $\theta \in \Im_{0}$, and applying the properties of exponential martingales, we evaluate the two components of the expectation. During the exercise repeated use is made of the independent increments property of Brownian motion. Evidently from the constraint (6), even though there are two apparent sources of randomness - from the stock price at two different points in time, $S_{\omega}\left(T_{n^{\star}}\right)$ and from $S_{\omega}\left(T_{n}\right)-$ a reduction ensues so that there is only one effective source of randomness. The first component of the expectation evaluates as:

$$
\begin{aligned}
E[ & \left.S^{*}\left(T_{n^{\star}}\right) \mathbf{1}_{\theta} \mid \Im_{0}\right] \\
= & \iint \mathbf{1}_{\theta} S_{\omega}^{*}\left(T_{n}\right) \mathrm{e}^{-\frac{1}{2} \sigma_{T_{\left[n, n^{\star}\right]}^{2}} \Delta T_{\left[n, n^{\star}\right]}} \\
& \times \mathrm{e}^{\sigma_{\left[n, n^{\star}\right]} \sqrt{\Delta T_{\left[n, n^{\star}\right]}} z^{\star}} \phi\left(z, z^{\star}\right) d z d z^{\star} \\
= & S_{0} \mathrm{e}^{-\frac{1}{2} \sigma_{\left[0, n^{\star}\right]}^{2} T_{n^{\star}}}\left[\int_{\mathbb{R}} \mathrm{e}^{\sigma_{[0, n]} \sqrt{T_{n}} z} \phi(z) d z\right] \\
& \times \int \mathbf{1}_{\theta} \mathrm{e}^{\sigma_{\left[n, n^{\star}\right]} \sqrt{\Delta T_{\left[n, n^{\star}\right]}} z^{\star}} \phi\left(z^{\star}\right) d z^{\star} \\
= & S_{0} \Phi\left(z_{+}\right) .
\end{aligned}
$$

This is the first term in the forward start value (4). Similarly the second component evaluates to:

$$
\begin{aligned}
E & {\left[S^{*}\left(T_{n}\right) \mathrm{e}^{-r \Delta T_{\left[n, n^{\star}\right]}} \mathbf{1}_{\theta} \mid \Im_{0}\right] } \\
= & \iint \mathbf{1}_{\theta} \mathrm{e}^{-r \Delta T_{\left[n, n^{\star}\right]}} S_{0} \mathrm{e}^{-\frac{1}{2} \sigma_{[0, n]}^{2} T_{n}} \\
& \quad \times \mathrm{e}^{\sigma_{[0, n]} \sqrt{T_{n}} z} \phi\left(z, z^{\star}\right) d z d z^{\star} \\
= & S_{0} \mathrm{e}^{-r \Delta T_{\left[n, n^{\star}\right]}} \mathrm{e}^{-\frac{1}{2} \sigma_{[0, n]}^{2} T_{n}} \\
= & \left.\times \int_{\mathbb{R}} \mathrm{e}^{\sigma_{[0, n]} \sqrt{T_{n}} z} \phi(z) d z\right] \int \mathbf{1}_{\theta} \phi\left(z^{\star}\right) d z^{\star} \\
= & S_{0} \mathrm{e}^{-r \Delta T_{\left[n, n^{\star}\right]}} \int \mathbf{1}_{\theta} \phi\left(z^{\star}\right) d z^{\star}
\end{aligned}
$$

which after multiplication by $x$ produces the last term in (4).

Unfortunately, no closed-form expression is applicable for a strike value that depends on more than one realization of equity price on the equity price path $(S(t), 0<t<T)$. For instance, even the relatively simple "strike weighted stock average", $X\left(S\left(T_{n}\right), S\left(T_{n-1}\right)\right) \equiv w S\left(T_{n-1}\right)+(1-$ w) $S\left(T_{n}\right), w>0$, already results in unmanageable bounds of integration when it comes to analytically evaluating the riskneutral expectation. Consequently, a claim that depends on several forward segments of volatility, such as $\max \left\{S\left(T_{n^{\star}}\right)-\right.$ $\left.X\left(S\left(T_{n}\right), S\left(T_{n-1}\right)\right), 0\right\}, n<n^{\star}$, can only be evaluated by two methods: (i) via simulation of equity paths or (ii) through numerical methods applied to the Black-Scholes partial differential equation. As the former choice is typically preferred, a fortiori, when running a simulation for pricing complex path dependent claims - be they American or European type - it is imperative to specify forward volatility correctly.

This is the simplest problem where components of the joint distribution of an equity price process are used, yet it still indicates the potential complexity of a "real world" valuation. The independent increments property of Brownian motion is repeatedly utilized in the derivation. It is not surprising, therefore, when the Brownan motion process is converted to geometric Brownian motion that the forward start valuation formula (4) does not depend on the immediate future. Using the simple formula, one can compare a sequence of forward start options, under varying volatility regimes, terminating at some time $t=T_{N_{k}}$ to the Black-Scholes value under the constant implied volatility assumption also expiring at $t=T_{N_{k}}$.

If more-complex contingent claims are to be evaluated, either European or American, where the valuation depends on properties of the joint distribution of prices, at several points in time, or across equities, an appeal can be made to 
simulation methods, e.g., projection methods of Longstaff and Schwartz (2001).

\section{FINISHING UP}

Even with the foreseeable available power of state-of-the art computing, dimension reduction is an important topic of research. The methods we are pursuing can be advantageous in at least two additional related areas. The first of these is with regard to Markov chain Monte-Carlo techniques. The fact that p.c. reduction consists of a set of orthogonal series permits the mixing of chains to proceed in a timely manner. The second relates to placing valuation in a context of portfolio optimization. Stochastic programming methods are powerful, but still suffer from the requirement that tens of millions of path generations are required (Ruszczynski and Shapiro 2003). Once again, the orthogonal nature of p.c. may permit the decomposition of a stochastic program into many constituent parts, each capable of being "solved" in a reasonable time period.

\section{REFERENCES}

Black, F., and M. Scholes. 1973. The pricing of options and corporate liabilities. Journal of Political Economy 81:637-659.

Cont, R., J. Da Fonseca, and V. Durrleman. 2002. Stochastic models of implied volatility surfaces. Economic Notes by Banca dei Paschi di Siena, SpA 31:361-377.

Cvitanić, J., and F. Zapatero. 2004. Economics and Mathematics of Financial Markets. MIT Press: Cambridge, Massachusetts.

Fengler, M. R., W. Härdle, and C. Villa. 2001. The dynamics of implied volatilities: A common principal components approach. SfB 373 Discussion Paper 2001, 38. Humboldt-Universität zu Berlin.

Flury, B. 1988. Common Principal Components and Related Methods. New York: John Wiley and Sons, Inc.

Flury, B., and W. Gautschi. 1986. An algorithm for simultaneous orthogonal transformation of several positive definite symmetric matrices to nearly diagonal form. SIAM Journal on Scientific and Statistical Computing 7:169-184.

Fouque, J-P., G. Papanicolaou, and K. R. Sircar. 2000. Derivatives in Financial Markets with Stochastic Volatility. Cambridge, United Kingdom: Cambridge University Press.

Härdle, W., and L. Simar. 2003. Applied Multivariate Statistical Analysis. Heidelberg: Springer-Verlag.

Harrison, M., and D. Kreps. 1979. Martingales and arbitrage in multiperiod securities markets. Journal of Economic Theory 20:381-408.

Harrison, M., and S. Pliska. 1981. Martingales and stochastic integrals in the theory of continuous trading.
Stochastic Processes and Their Applications 11:215260.

Hsieh, W. W. 2004. From principal component analysis (PCA) to nonlinear PCA. AMS Short Course 9:297325.

Ihaka, R., and R. Gentleman. 1996. R: A language for data analysis and graphics. Journal of Computational and Graphical Statistics 5:299-314.

Longstaff, F. A., and E. S. Schwartz. 2001. Valuing American options by simulation: A simple least-squares approach. Review of Financial Studies 14:113-147.

Margrabe, W. 1978. The value of an option to exchange one asset for another. Journal of Finance 33:177-86.

Merton, R. 1971. Theory of rational option pricing. Bell Journal of Economics and Management 4:141-183.

Øksendal, B. 2003. Stochastic Differential Equations An Introduction with Applications. Heidelberg: SpringerVerlag.

Pelsser, A. 2000. Efficient Methods for Valuing Interest Rate Derivatives. London: Springer-Verlag.

R Development Core Team. 2004. R: A Language and Environment for Statistical Computing, Vienna, Austria: R Foundation for Statistical Computing. ISBN 3900051-00-3, <http://Www.R-project.org> [Accessed on August 24, 2004].

Ruszczynski, A., and A. Shapiro, eds. 2003. Stochastic Programming, Handbook in Operations Research and Management Science, Amsterdam: Elsevier.

Wharton Research Data Services. Ivy-DB OptionMetrics. <http: / /www. optionmetrics. com> [Accessed on August 24, 2004].

\section{AUTHOR BIOGRAPHIES}

DAVID GOLDSMAN is a Professor in the School of Industrial and Systems Engineering at the Georgia Institute of Technology. He received his Ph.D. in Operations Research and Industrial Engineering from Cornell University. His research interests include simulation output analysis and ranking and selection. He has served as the Simulation Department Editor of IIE Transactions and an Associate Editor for SCS Transactions. He is an active participant in the Winter Simulation Conference, having been Program Chair in 1995, and having served on the WSC Board of Directors since 2002. His e-mail address is <sman@isye.gatech. edu>, and his web page is <www. isye.gatech. edu/ ${ }^{\sim} \operatorname{sman}>$.

RAY POPOVIC is working on his Ph.D. thesis in the area of Quantitative and Computational Finance, School of Industrial and Systems Engineering at the Georgia Institute of Technology. His primary interests lie in calibration methods applied to contingent claim valuation and hedging of equity and fixed income markets. His e-mail address is <raypopovic@gmail.com>. 